\title{
In situ Study of the Order-Disorder Transformation in Lithium Ferrite
}

\author{
Omer Van der Biest ${ }^{1}$, Gareth Thomas ${ }^{2 \dagger}$ \\ 1. Department of Materials Engineering (MTM), K U Leuven, 3001 Leuven, Belgium \\ 2. Department of Materials Science and Engineering, University of California, Berkeley, CA 94720
}

The lithium ferrite of interest here has as formula $\mathrm{LiFe}_{5} \mathrm{O}_{8}$. The structure of this compound has been reported by Braun [1]. It has an inverse spinel structure (space group $\mathrm{Fd} 3 \mathrm{~m}$ ) with the allowed tetrahedral sites filled with $\mathrm{Fe}^{3+}$ and the allowed octahedral sites occupied by a 3:1 mixture of iron and lithium ions. Above $750{ }^{\circ} \mathrm{C}$ these ions take on a random arrangement. Below this critical temperature, the ordered structure can occur in two enantiomorphous forms, $\mathrm{P} 4_{1} 32$ (right handed screw axis) and $\mathrm{P} 4332$ (left handed screw axis). Hence in the ordered structure the boundaries between ordered domains can consist of the normal antiphase boundaries (APB) which are characterized by a translation vector when the space group does not change across the boundary. In addition domain boundaries can also include enantiomorphous boundaries which are characterized by a mirror symmetry operation, or a combination of mirroring and translation [3].

The study reported here was done at the high voltage electron microscopy facility which was available at Imperial College London in 1975. The microscope was equipped with an environmental hot cell. The aperture type environmental cell allowed to maintain an oxygen partial pressure of about $5 \mathrm{kNm}^{-2}$ around the specimen. This prevented undesirable reduction reactions to occur by reaction of the oxide at high temperatures with the residual hydrogen in the microscope atmosphere. Such a reaction in a hot stage examination of phase transformations in cobalt ferrite was observed by De Jonghe and Thomas [2], resulting in oxygen loss from the specimen and decomposition to cobalt metal and an iron-rich spinel. No such reduction was observed in the experiments reported on here. The lithium ferrite samples were in the form of single crystal discs which had been chemically polished to perforation in hot phosphoric acid.

Studies of the dynamics of the order-disorder reaction were made by a technique of dark-field imaging a superlattice reflection which enabled the nucleation of well-defined ordered nuclei growing in a disordered matrix to be observed and recorded photographically via TV and video tape equipment. Rapid changes in the state of order of the specimen on either heating or cooling through the critical ordering temperature $\left(\sim 750{ }^{\circ} \mathrm{C}\right)$ were registered. Figure 1 shows some selected frames from such a sequence. Ordering was observed to proceed by the homogeneous nucleation and growth of spherical ordered domains whose growth was perfectly constant until they impinged to form boundaries (APB or enantiomorphous boundaries). Hence this disorder to order reaction is clearly a first order phase transition as the order parameter changes discontinuously at the domain boundaries. Increasing the amount of undercooling increased the nucleation rate and produced a maximum in the growth process.

Upon heating the material in the ordered state, disordering proceeded first at the boundaries between the ordered domains. Disordering on heating was observed to occur rapidly by the APB / enantiomorphous boundaries broadening to form disordered layers isolating the ordered domains. These domains then either continued to shrink in size or else their contrast faded uniformly as they disordered. 
Due to circumstances, these results here were never published in a refereed publication. However a 16 $\mathrm{mm}$ movie with sound has been produced and will be presented at the meeting. Commentator on the sound track is Prof. Gareth Thomas. The movie will be made available on the internet because it has also educational value.

\section{References:}

[1] P.B. Braun, Nature, Lond, 170, (1952) p 1123.

[2] L.C. De Jonghe and G. Thomas Mater. Sci. Eng 8 (1971) p 259

[3 ] O. Van der Biest and G. Thomas, Acta Cryst. A31, (1975) p70-76
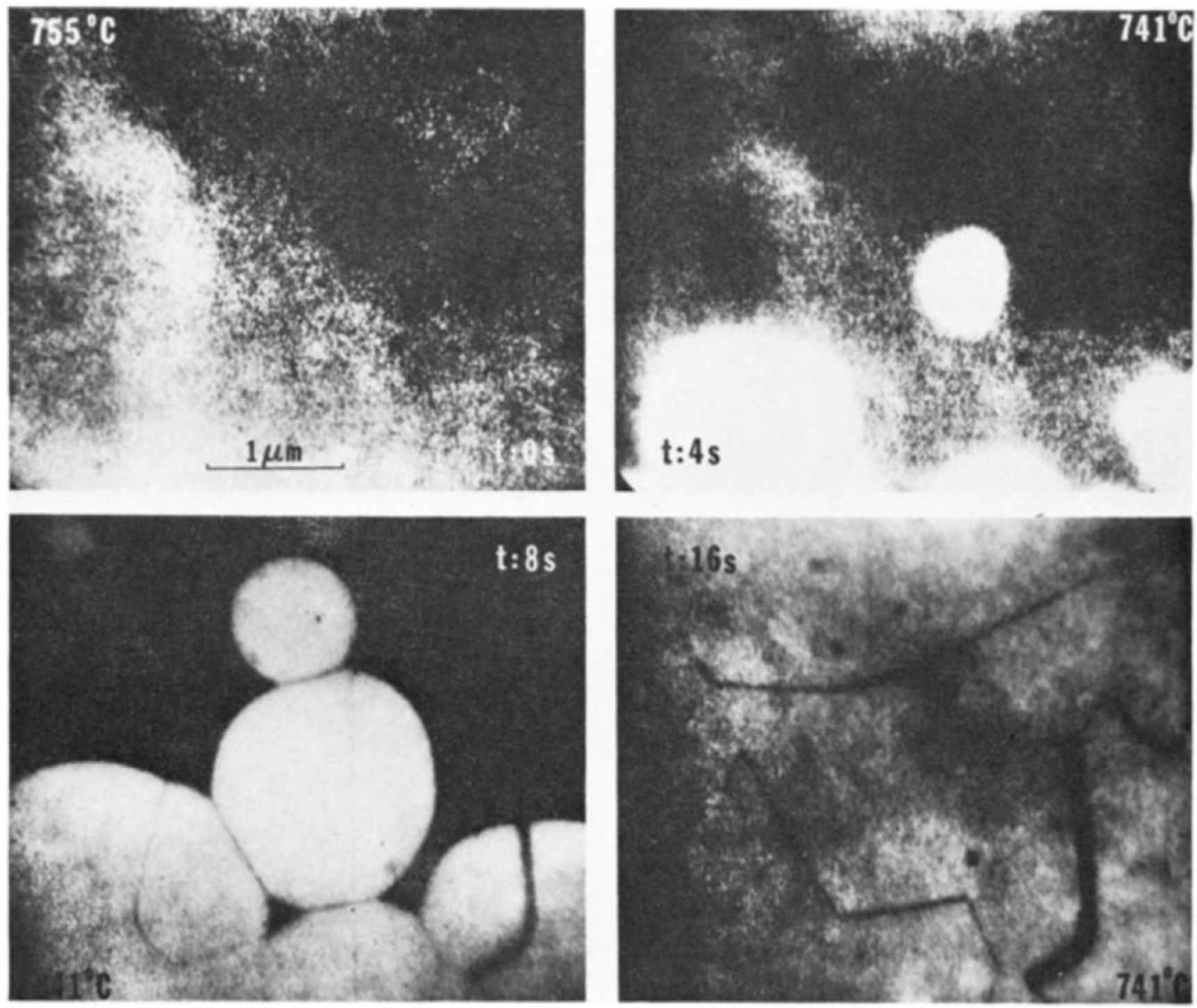

Figure 1. Observations of the disorder $\rightarrow$ order transformation in lithium ferrite while cooling the sample from above the critical temperature $\left(\sim 750^{\circ} \mathrm{C}\right)$ to just below it. Images are formed with a 112 superlattice reflection at $1 \mathrm{MV}$ and are for the same area shown as a function of time. The sample is also under a partial pressure of oxygen of $\sim 5 \mathrm{kNm}^{-2}$ (40 Torr). The nucleation and growth of ordered domains (bright) from the disordered state (dark background) is clearly observed. 